\title{
Rol de la Economía Social y la Universidad en orden a un emprendimiento basado en el conocimiento tecnológico y los valores
}

\author{
Juan Francisco Juliá Igual \\ Elena Meliá Martí \\ Eduardo Miranda Ribera
}

RESUMEN: Hoy en día se reconocen las sociedades más desarrolladas por contar con un emprendimiento innovador que hace un uso intensivo del conocimiento y utiliza las tecnologías más avanzadas. Estas sociedades, si además de economías competitivas pretenden ser sociedades más justas, solidarias y sostenibles deben también emprender con valores que les conduzcan a ello. El presente trabajo pretende abordar la necesidad de avanzar en una economía más intensiva en conocimiento, especialmente tecnológico, que permita afrontar el reto que supone la llamada $4^{a}$ revolución tecnológica, contribuyendo al desarrollo de una sociedad más competitiva y al mismo tiempo con valores. Así, este trabajo realiza una revisión de la realidad de la universidad española, y de su papel ante el reto tecnológico. Además, efectúa un análisis de la presencia y peso de la economía social en aquellos sectores más identificados con la llamada cuarta revolución industrial, y establece que no existen diferencias de ámbito económico-financiero que puedan explicar la previamente constatada menor presencia de las fórmulas de economía social frente a las de capital. De la revisión y el análisis efectuado, este trabajo concluye que para que se avance en esta economía competitiva y con valores, es crucial el papel de la universidad, fomentando y desarrollando un emprendimiento innovador, inteligente y con valores. En las últimas cuatro décadas la Economía Social ha experimentado un importante avance en términos cuantitativos y cualitativos, y también lo ha hecho el emprendimiento, en particular, el universitario, contribuyendo a avanzar en la generación de una economía con mayor uso del conocimiento y, sobre todo, con valores. Sin embargo, continúa siendo necesario impulsar el uso de fórmulas de Economía Social en el emprendimiento derivado del ecosistema universitario, y con ello su grado de presencia en los sectores tecnológicos más avanzados, y en particular en los más estrechamente vinculados a la llamada cuarta revolución industrial.

PALABRAS CLAVE: Economía Social, emprendimiento, Universidad, conocimiento, revolución tecnológica.

CLAVES ECONLIT: A20, 129, 135.

Cómo citar este artículo / How to cite this article: JULIÁ, J.F., MELIÁ, E. \& MIRANDA, E. (2020): "Rol de la economía social y la universidad en orden a un emprendimiento basado en el conocimiento tecnológico y los valores", CIRIEC-España, Revista de Economía Pública, Social y Cooperativa, 98, 31-57. DOI: 107203/ CIRIEC-E.98.15905.

Correspondencia: Juan F. Juliá Igual, ORCID: 0000-0003-4667-1865; Elena Meliá Martí, ORCID: 00000002-0417-2139, y Eduardo Miranda Ribera, ORCID: 0000-0003-3833-6645. CEGEA-Universitat Politècnica de València, Campus Vera s/n, 46023, Valencia. E-mail de contacto: jjullia@esp.upv.es. 


\section{EXPANDED ABSTRACT}

\section{Role of the Social Economy and the University in order to a venture based on technological knowle- dge and values}

\section{Objectives}

The present work aims to study the need for a more knowledge-intensive economy, especially technologically based, that allows to face the challenge of the so-called 4th technological revolution, contributing to the development of a more competitive society as well as with values. Thus, this work aims to do a revision of the reality of the spanish university and its role facing the technological challenge. In addition, it aims to make an analysis of the presence and weight of the social economy in the sectors most identified with the so-called fourth industrial revolution, to establish if there are economic and financial differences that explain the higher presence of the investor owned firms versus social economy enterprises.

\section{Design and methodology}

In addition to the literature revision, we have used SABI database, in order to identify social economy companies that operate in technological sectors, specifically those related to digital economy and the fourth industrial revolution. Financial and economic variables of both have been analyzed and compared.

\section{Results and conclusions}

From the literature review and the analysis carried out, this work concludes that the presence of social economy companies in technological sectors related to the digital economy is smaller than the one of the investor owned companies, and in addition this presence is decreasing over the years. On the other hand, there are no differences in their economic and financial indicators that support a greater preference for investor owned enterprises capital compared to social economy enterprises.

We conclude that in order to advance in this competitive economy with values, the role of the university is crucial, fostering and developing an innovative, intelligent and with values entrepreneurship. In the last four decades the social economy has undergone significant progress in quantitative and qualitative terms, and so has entrepreneurship. However, it is still necessary to promote the use of social economy formulas in entrepreneurship derived from the university ecosystem, and with it, its presence in the most advanced technological sectors, and in particular those most closely linked to the so-called fourth industrial revolution.

KEYWORDS: Social economy, entrepreneurship, University, knowledge, technological revolution. 


\section{Introducción y objetivos'}

El presente trabajo pretende abordar la necesidad de avanzar en una Economía más intensiva en conocimiento, especialmente tecnológico que permita afrontar el reto que supone la llamada $4^{\mathrm{a}}$ revolución tecnológica, contribuyendo al desarrollo de una sociedad más competitiva y al mismo tiempo que ello se realice desde una economía con valores, mostrando, por un lado, cómo la Universidad constituye un ecosistema muy favorable a un emprendimiento innovador y tecnológicamente desarrollado como la Economía Social, fórmula cuyos principios y valores la convierten en un instrumento de interés, para un emprendimiento más social.

El marco teórico de este trabajo aborda la realidad de sistema universitario español, así como su contribución al reto tecnológico.

A partir de este punto, el objeto del trabajo es en primer lugar analizar la presencia y peso de la Economía Social en aquellos sectores más identificados con la llamada cuarta revolución industrial. En segundo lugar, establecer si existen diferencias en el ámbito económico-financiero que puedan explicar la mayor o menos presencia de unas fórmulas frente a otras (de capital frente a las de economía social). Por último, se realiza una discusión hacia el necesario avance hacia una economía del conocimiento y con valores, garante de un mayor progreso social. Acerca de cómo el uso más intensivo del conocimiento se corresponde con economías más competitivas, siendo las Universidades entes clave en la generación y transmisión de conocimiento. Y de la necesidad de que la universidad desempeñe un papel más activo, a la hora de dar a conocer las fórmulas empresariales de economía social, y su capacidad de desarrollar su actividad exitosamente también en sectores de tecnologías avanzadas, con un uso intensivo del conocimiento. En este camino, mostraremos que el binomio conformado por la llamada Economía Social y la Universidad constituyen los elementos clave para propiciar una Economía inteligente y con valores.

El trabajo aborda en los epígrafes 2 y 3 la situación del sistema universitario español y su papel ante el reto tecnológico. En el epígrafe 4 se realiza un análisis del peso de las empresas de economía social y el emprendimiento en sectores de carácter tecnológico dentro de la economía digital. En el epígrafe 5 se analizan las diferencias de carácter económico-financiero en las empresas de estos sectores creadas bajo fórmulas de capital y de economía social. Por último, en los epígrafes 6 y 7 se aborda la discusión y las conclusiones.

1.- Este trabajo parte de uno anterior de dos de los autores referido a la Economía Social y la Constitución española, incorporando en este caso el estudio sobre el grado de presencia de las empresas de Economía Social en el conjunto de compañías cuyas actividades se corresponden de forma más visible con la llamada cuarta revolución industrial. 


\section{El sistema universitario español}

La dimensión social de la Universidad debe hoy necesariamente ser entendida en un sentido amplio, desde su visión tradicional de institución al servicio de la sociedad que debe ayudar a dar respuesta adecuada a sus principales demandas sociales a partir de la generación de conocimiento y su transmisión. Este servicio a la sociedad debe dirigirse, en primer lugar, a los ciudadanos y, en particular, a los estudiantes, pero también a todos los agentes sociales, como instituciones y organizaciones que los representan, y cómo no al tejido productivo y sus empresas.

Actualmente, todos estos colectivos entienden y comparten la necesidad de incorporar más conocimiento en el desarrollo de las distintas actividades económicas y servicios sociales, lo que pone de relieve el papel motor que adquiere la Universidad como elemento de transformación. Por ello, se reclama un compromiso cada vez más activo de la Universidad en su misión docente e investigadora, en orden a valorizar el conocimiento también mediante la promoción del emprendimiento y la innovación. La idea de un crecimiento basado en el conocimiento integrador y sostenible requiere de la mayor extensión de la actividad universitaria, siendo necesaria una Universidad más accesible, que provea una mayor igualdad de oportunidades y evite la pérdida de talento.

En este sentido, la Universidad española ha venido creciendo desde la promulgación de la Constitución Española y, en particular, con su desarrollo normativo al amparo del nuevo marco constitucional como fue en su día la Ley de Reforma Universitaria, que supuso un importante avance. La extensión territorial del mapa universitario desde entonces ha permitido duplicar el número de universitarios y ha significado que, en España, cualquier estudiante tenga acceso a una Universidad en un radio no mayor a $50 \mathrm{~km}$ desde su residencia. Ello ha contribuido sin ninguna duda a una mayor cohesión social y territorial (Pérez Hernández, 2018).

La preocupación por una necesaria mejora de la calidad debe ir más allá de colocar unas pocas Universidades en los primeros lugares de los rankings internacionales, debiendo por el contrario abarcar a todo el sistema universitario, permitiendo la extensión de un servicio universitario de calidad al conjunto de la población. Así, en una comunicación del Foro Económico Mundial se afirmaba en relación con lo que esperan los ciudadanos de la Universidad que, "lo más importante para el ciudadano promedio de un país no es si tiene una Universidad como Stanford o Oxford, sino más bien, la calidad de la Universidad a la que probablemente sus hijos asistan" (Chan, 2017).

Ciertamente, para avanzar en igualdad de oportunidades y equidad social y territorial, debemos velar por la suficiente amplitud de un sistema universitario de calidad que permita poner en valor todo 
el talento de nuestros jóvenes, con independencia de su nivel social y ubicación territorial (Sánchez et al, 2018; Bel y Lejarriaga, 2018). Igualmente, se puede afirmar que actualmente contamos con sistema universitario de calidad razonable, si analizamos los principales indicadores que nos hablan del desempeño del conjunto de nuestras universidades. En efecto, tanto si vemos el posicionamiento de nuestras universidades en los rankings internacionales más reconocidos, como si atendemos a la evolución del rendimiento docente y la producción científica de nuestros campus, no podemos más que emitir una valoración positiva de la actividad académica desarrollada por nuestras instituciones (Julia, 2019).

Así, si nos referimos a la posición que ocupan nuestras Universidades en los tres principales rankings internacionales (ARWU, THE y QS), aún no situando ninguna entre las 100 mejores, sí observamos que contamos con 45 Universidades (43 públicas y 2 privadas) que aparecen en alguno de estos rankings entre las 1.000 mejores en 2019 (38 en ARWU, 37 en THE y 25 en QS). Y esto, si tenemos en cuenta que en el mundo existen más de 20.000 Universidades, significa que 4 de cada 5 Universidades públicas españolas están en el rango del $5 \%$ de las mejores instituciones académicas del mundo.

Otro dato que testimonia la extensión de un sistema universitario de razonable calidad es que, si nos comparamos con las mayores economías del mundo, algunas de las cuales cuentan con varias Universidades en los primeros puestos de los rankings, solo el Reino Unido, de entre las 10 mayores economías del mundo, ofrece una ratio de Universidades entre las mil mejores por millón de habitantes superior a España. Así, por ejemplo, mientras que España cuenta con una Universidad entre las 1.000 mejores por cada 1.200.000 habitantes, Estados Unidos cuenta con 1 por cada 1.500.000. Además, si hablamos de algunos ámbitos del conocimiento y disciplinas, España cuenta con una veintena de Universidades que se sitúan entre las 50 mejores del mundo (Delgado, 2017).

Estos rankings internacionales ofrecen una visión de la actividad Universitaria que no es completa, ya que más bien, está relacionada con su producción científica y notoriedad académica (ARWU), a la que incorporan también indicadores de orden reputacional (THE, QS), por lo que algunos de estos rankings están elaborando otros listados con el objetivo de poder medir el rendimiento docente, empleabilidad o compromiso social.

Así, si nos centramos en la función docente y su contribución a la empleabilidad de los egresados, podemos observar como el THE en 2018 presentó el Europe Teaching Ránking, en el que aparecían 42 Universidades españolas entre las 242 mejores de Europa, 4 de ellas entre las 100 mejores, si bien es cierto que este ránking, en esta su primera edición, contempla solo 8 países de Europa, las 5 mayores potencias (Alemania, Reino Unido, Francia, Italia, España), a las que se unen Holanda, Portugal e Irlanda. Por otro lado, el QS Employability, centrado en la contribución a la empleabilidad que deriva del desempeño académico de las Universidades, y que cuenta entre sus indicadores con la opinión de los empleadores, incluía en el 2020 a 15 Universidades españolas entre las 500 mejores 
del mundo, 4 de ellas entre las 100 mejores, lo que situaba al sector universitario español como el tercero en Europa en relación con su tamaño de población.

Estos datos permiten afirmar que el desempeño docente de la Universidad española es bueno, y su rendimiento es más que aceptable cuando nuestro gasto en educación superior es todavía un $20 \%$ inferior, en porcentaje del PIB, al de la media de la OCDE. Estos datos contradicen algunas opiniones que, en sentido contrario, se han mantenido en algún momento acerca del desempeño académico de las Universidades españolas. Del mismo modo, si hablamos de producción científica debemos hacer un balance positivo, ya que España se ha consolidado en los últimos años entre la décima y undécima posición mundial, con algo más del 3,3\% de la producción científica mundial en número de publicaciones, de forma similar a la posición a la que ha obtenido por número de investigadores más altamente citados, décima en el mundo en 2018.

Ello no quiere decir que no se pueda y deba mejorar, y que no haya áreas de clara mejora. Si nos fijamos en la transferencia de conocimiento, elemento clave para valorizarlo al hacerlo útil para el tejido productivo, nos encontramos con indicadores que no se corresponden con nuestro nivel de desempeño docente e investigador, y que comparativamente nos alejan de algunos de los países más avanzados. A modo de ejemplo, si hablamos de registro de patentes vemos que nuestro peso a nivel mundial en patentes internacionales es tan solo de 0,6\%, ocupando la posición 18 según la Oficina Europea de Patentes y Marcas (Julia, 2019).

Es evidente que desde esta perspectiva se puede hablar del potencial que tiene nuestro sistema universitario, y de cómo en los últimos años ha contribuido a una mayor igualdad de oportunidades y a una mayor cohesión social y territorial, pero, también, de la necesidad de contribuir de una forma más activa y efectiva a la valorización de conocimiento (Piriz, 2018). Es necesaria una visión de la dimensión social de la Universidad más amplia, que incorpore la transferencia, el emprendimiento y la innovación como una parte esencial de la misma.

\section{La Universidad española y el reto tecnológico}

En un momento en el que nos encontramos inmersos en la llamada cuarta revolución industrial que supone el dominio de la denominada sociedad digital, en todas y cada una de las actividades, es bien claro, que ello obliga a afrontar un importante reto tecnológico para aquellas economías que pretenden ser avanzadas, y se hace necesario el concurso de actividades de una mayor intensidad tecnológica y científica poniendo en valor las disciplinas conocidas bajo las siglas de STEM (Ciencia, 
Tecnología, Ingeniería y Matemáticas), de manera que, se deberá contar con el suficiente capital humano y el adecuado nivel de formación. Algunos autores destacan la necesidad de un sistema educativo, en el que no solo se formen perfiles tecnológicos en estas disciplinas, sino que también deberán aportar capacidades creativas para adaptarse a los constantes cambios, que requerirán de tecnología y creatividad. De este modo, cabe hablar también de capacidades STEAM donde la "A" representa lo relacionado con el arte y la creatividad (González-Páramo, 2017).

Un reto tecnológico como el que supone tan intensa transformación digital en nuestra actividad productiva requerirá de jóvenes bien formados y con capacidades en todas estas disciplinas, algo que justifica la preocupación por la demanda de estudios en las mismas, de forma muy especial en el caso de las Ingenierías, pero también del conjunto de disciplinas que conforman las STEM. En el caso de España esta preocupación es importante dado que se ha producido un descenso muy importante en la demanda de estos estudios ya que mientras que en el año 2006 el conjunto de estudiantes que demandaba este tipo de estudios representaba el 30,5\% del alumnado, en el año 2018 tan solo era el $24 \%$, y apenas un $14 \%$ si nos referimos a ingeniera informática, lo que contrasta con las necesidades del tejido productivo que según un informe de ADECCO se cifran en un $37,1 \%$ del porcentaje de ofertas de empleo (Aldás, 2019).

La gravedad de este descenso en la demanda de este tipo de estudios frente a la creciente necesidad de este tipo de perfiles es mayor en el caso de nuestro país si observamos las diferencias en cuanto al perfil de demanda de estos estudios en la Unión Europea y España donde el porcentaje es más de 4 puntos inferior y con una mayor diferencia con respecto a países como Alemania o Reino Unido. Algunas de las razones que pretenden explicar esta situación vienen relacionadas con la existencia de dos importantes brechas en lo referente a los perfiles del tipo de estudiante que accede a estos estudios. La primera de ellas es la brecha de género, que también se da en otros países, y que en España es muy acusada, especialmente en las ingenierías, donde apenas se alcanza el 25\% de mujeres entre sus estudiantes, y de forma aún más intensa en los estudios de informática, pero también cabe hablar de una segunda brecha relacionada con el nivel de renta de las familias de los estudiantes ya que presumiblemente la menor tasa de rendimiento académica tradicional de estos estudios contribuya a que los estudiantes de familias con menos rentas que necesitan de un mejor expediente académico para acceder a becas y ayudas al estudio vean sus expectativas disminuidas si acceden a este tipo de estudios, que como apuntamos cuentan en general con un peor rendimiento académico.

Por otro lado, si bien los datos confirman que desde el punto de vista laboral las expectativas de acceso al empleo como en el futuro de ingresos salariales son mayores para quienes cursan estos estudios, como muestran los datos sobre inserción laboral de egresados universitarios y evolución de sus ingresos salariales, no parece que la percepción de los estudiantes que deciden inclinarse por otros estudios sea esa. Algo que se corresponde con el éxodo de estudiantes de estas disciplinas tecnológicas hacia otros países donde su inserción laboral y nivel retributivo es mayor, como países 
como Alemania y Reino Unido donde paradójicamente cuentan con un mayor porcentaje de sus estudiantes que se inclinan por estas disciplinas (Pastor, 2019).

En este contexto, es del mayor interés la promoción de actividades emprendedoras relacionadas con el uso de nuevas tecnologías, que valoricen nuestro conocimiento y capital humano en este campo (Palomo et al, 2018). Las Universidades están sin duda desarrollando ya una intensa labor en esta dirección acompañadas por instituciones como Universia y Santander Universidades, creando oficinas y estructuras que promueven y ayudan al desarrollo de un verdadero ecosistema emprendedor en los campos universitarios y que todo parece apuntar que favorece un emprendimiento de calidad en términos de capacidad innovadora y desarrollo tecnológico, para un mejor posicionamiento ante el reto que supone la revolución tecnológica.

Efectivamente, el Centro Internacional Santander Emprendimiento entre cuyos objetivos esta la promoción de este tipo de emprendimientos mostraba en una reciente publicación como comparativamente el emprendimiento que emerge del ecosistema universitario, muestra no solo ya una tasa de actividad significativa según las encuestas Global Entrepreneurship Monitor (GEM) sino que también se distingue por su mayor calidad en términos de innovación uso de tecnologías avanzadas, internacionalización de negocio e incluso un menor índice de microemprendimiento, lo que puede contribuir en un futuro a conformar un tejido empresarial más preparado para lo que significa el reto tecnológico de la cuarta revolución (Julia et al, 2015).

\section{Emprendimiento bajo sus fórmulas en la economía digital}

La economía social ha contado y cuenta en España con un peso relevante en la economía. No en vano, España fue el primer país europeo en promulgar una norma para esta forma de economía, algo que luego han hecho otros países europeos (Monzón y Chaves, 2018), siendo en la actualidad el quinto país de Europa por empleo remunerado en economía social, como se observa en la figura 1 (Monzón y Chaves, 2018), y el noveno mundial según un reciente informe del Secretariado de Naciones Unidas (Dave Grace, 2014).

Sin embargo, y pese a que esta fórmula empresarial está muy presente en algunos sectores tradicionales de actividad, como son el agroalimentario, queda por demostrar que esté teniendo una presencia similar en los emprendimientos de corte tecnológico, y constatados en el apartado anterior como claves del futuro de la economía. 
Así, si bien es cierto que el emprendimiento y la innovación se han incrementado en los campus universitarios, como ya hemos visto, no es menos cierto que esto no ha sido generalmente bajo figuras de la Economía Social, en las que los principios y valores forman parte de su práctica económica, y en cuyos modelos económicos lo social adquiere especial relevancia (Juliá et al. 2014; Seguí et al 2017).

Figura 1. El empleo de la economía social

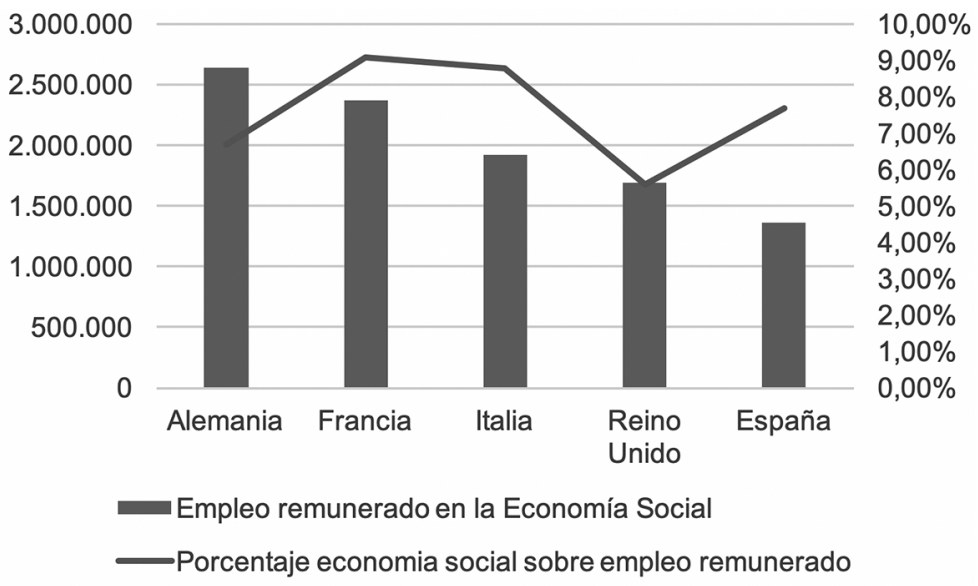

FUENTE: Elaboración propia a partir de CIRIEC-España 2018.

Al efecto de visualizar cual es la presencia y peso de la economía social en las empresas de carácter tecnológico dentro de la economía digital, se ha efectuado un trabajo de consulta y análisis en la base de datos $\mathrm{SABI}$ (tabla 1). Esta base de datos incluye información jurídico-económico-financiera de más de 2 millones de empresas españolas, siendo muy utilizada en investigación por incluir a un porcentaje muy elevado y representativo de las empresas que operan en el territorio español ( $78 \%$ en sociedades anónimas, $65 \%$ de las sociedades laborales y $31 \%$ de las cooperativas) (tabla 1). 


\section{Tabla 1. Alcance de la base de datos SABI en la economía em- presarial española}

\begin{tabular}{|c|c|c|c|c|}
\hline & $\begin{array}{l}\text { Nº de Empresas } \\
\text { activas según el } \\
\text { DIRCE (2018) }\end{array}$ & \multicolumn{2}{|c|}{$\mathrm{N}^{\circ}$ de empresas incluidas en $\mathrm{SABI}$} & \multirow{2}{*}{$\begin{array}{c}\text { \% empresas activas en SABI } \\
\text { / empresas activas según } \\
\text { DIRCE }\end{array}$} \\
\hline & & Totales & Activas & \\
\hline SA & 78.866 & 127.653 & 61.336 & $78 \%$ \\
\hline SL & 1.181 .391 & 1.508 .100 & 771.427 & $65 \%$ \\
\hline SCoop & 20.707 & 8.027 & 6.341 & $31 \%$ \\
\hline
\end{tabular}

FUENTE: Elaboración propia a partir del Directorio Central de Empresas, obtenida a partir de la base de datos del INE (INEbase), y de la base de datos SABI.

A la hora de establecer los sectores en los que se ha centrado la consulta, hemos optado por seleccionar aquellos altamente innovadores, y que sustentan su actividad bien en los servicios de carácter informático o en las llamadas Tecnologías de la Información y la Comunicación (TIC), por ser sectores clave sobre los que sin duda pivotará la que se ha venido a llamar $4^{a}$ revolución industrial. Con este objeto, hemos seleccionado, a partir de la Clasificación Nacional de Actividades Económicas (CNAE), los siguientes sectores y subsectores de actividad:

- CNAE 26: Fabricación de productos informáticos, electrónicos y ópticos,

- CNAE 61: Telecomunicaciones,

- CNAE 62: Programación, consultoría y otras actividades relacionadas con la informática,

- $\quad$ CNAE 631: Proceso de datos, hosting y actividades relacionadas; portales web

Hay que apuntar que esta selección no incluye todas las empresas con actividad tecnológicamente avanzada, ya que hay empresas con actividad principal o secundaria declarada en otros sectores, como es el caso de la "fabricación de maquinaria", o en algunos casos "fabricación de material" que operan en el ámbito de la robótica y de las máquinas inteligentes, o en otros ámbitos como el de la biotecnología o bioingeniería, cuya actividad tiene un alto componente tecnológico.

A partir de la información obtenida, podemos señalar que el peso de la economía social en las empresas que operan con tecnologías de la Información y la Comunicación (TIC) o con tecnologías Informáticas (IT), en adelante IT-TIC, es inferior al que encontramos en el conjunto de la economía (solo un 1,62\% de las empresas frente al 1,95\% del conjunto de la economía). Sin embargo, conviene matizar que este menor peso de la economía social no se da de forma generalizada en todas sus formas particulares, siendo la cooperativa la forma de economía social que se muestra con menos 
peso $(0,19 \%$ en sectores IT-TIC frente a un $0,52 \%$ en la generalidad de los sectores). Por el contrario, las sociedades anónimas laborales (SAL) y sociedades limitadas laborales (SLL) no se encuentran en inferioridad, sino que tienen un diferencial positivo con respecto a la generalidad de los sectores (las SLL pasan del $1,21 \%$ al $1,26 \%$ y las SAL pasan del $0,10 \%$ al $0,12 \%$ ).

\section{Tabla 2. Peso de la economía social en los sectores IT-TIC: in- formático, electrónico, de telecomunicaciones, de programa- ción y de proceso de datos (CNAEs 26, 61, 62, 631)}

\begin{tabular}{|l|c|c|c|c|}
\hline & \multicolumn{2}{|c|}{$\begin{array}{c}\text { No de empresas en todos los sectores de } \\
\text { la economía }\end{array}$} & $\begin{array}{c}\text { No de empresas en sectores tecnológicos } \\
\text { (CNAEs 26, 61, 62, 631) }\end{array}$ \\
\hline SA (excl SAL) & 87.194 & $6,38 \%$ & 2.106 & $5,85 \%$ \\
\hline SL (excl SLL) & 1.248 .328 & $91,32 \%$ & 32.250 & $89,62 \%$ \\
\hline FOMAS DE E. SOCIAL & 26.602 & $1,95 \%$ & 583 & $1,62 \%$ \\
\hline - SAL & 1.338 & $0,10 \%$ & 43 & $0,12 \%$ \\
\hline - SLL & 16.607 & $1,21 \%$ & 454 & $1,26 \%$ \\
\hline - ASOCIACION & 1.556 & $0,11 \%$ & 18 & $0,05 \%$ \\
\hline - SCOOP & 7.101 & $0,52 \%$ & 68 & $0,19 \%$ \\
\hline OFJUR & 4.923 & $0,36 \%$ & 1.047 & $2,91 \%$ \\
\hline TOTAL & 1.367 .047 & $100,00 \%$ & 35.986 & $100,00 \%$ \\
\hline
\end{tabular}

SA: Sociedades anónimas; SL: sociedades limitadas, excluidas las laborales; SCOOP: sociedades cooperativas; SAL Y SLL: sociedades anónimas laborales y sociedades limitadas laborales. OFJUR: otras formas jurídicas. FUENTE: Datos extraídos de SABI, en septiembre de 2019, seleccionando solo las empresas activas, y en el caso de los sectores tecnológicos, aquellas que incluyen los códigos CNAE indicados como primario o secundario.

Si observamos las estadísticas de creación de empresas de capital y de economía social para la generalidad de los sectores, y las de los sectores IT-TIC, obtenidas de la base de datos SABI, se observan datos interesantes (Tabla 3): 
Tabla 3. Sociedades creadas en España desde el año 2000 al 2017 (de todos los sectores de actividad)

\begin{tabular}{|l|c|c|c|c|}
\hline & \multicolumn{2}{|c|}{ Según el INE } & \multicolumn{2}{c|}{ Según el Ministerio de Trabajo } \\
\hline & SAy SL & S COOP & SCOOP & SLAB \\
\hline $\mathbf{2 0 0 0 - 2 0 0 4}$ & $21.3 \%$ & $29.6 \%$ & $41.1 \%$ & $59.8 \%$ \\
\hline $\mathbf{2 0 0 5 - 2 0 0 9}$ & $29.3 \%$ & $29.7 \%$ & $23.1 \%$ & $25.6 \%$ \\
\hline $\mathbf{2 0 1 0 - 2 0 1 4}$ & $30.8 \%$ & $25.9 \%$ & $20.8 \%$ & $11.7 \%$ \\
\hline $\mathbf{2 0 1 5 - 2 0 1 7}$ & $18.6 \%$ & $14.8 \%$ & $15.1 \%$ & $2.9 \%$ \\
\hline Total & $100.0 \%$ & $100.0 \%$ & $100.0 \%$ & $100.0 \%$ \\
\hline
\end{tabular}

SA: Sociedades anónimas; SL: sociedades limitadas, excluidas las laborales; SCOOP: sociedades cooperativas; SLAB: sociedades laborales

FUENTE: INE y Ministerio de Trabajo, Migraciones y Seguridad Social.

Lo primero que se observa del análisis de los datos (tablas 3 y 4), es que la constitución de sociedades cooperativas y laborales de corte IT-TIC en el periodo 2000-2017 es decreciente, estando muy concentrada su creación en el primer lustro (2000-2004), que integra el 56\% de las Sociedades laborales creadas y el $45,9 \%$ de las cooperativas (tabla 4). A medida que avanzamos en el tiempo, la tasa de creación de empresas de economía social decrece, frente a la de las sociedades anónimas y limitadas, cuya creación tiene un ritmo mucho más equilibrado en el tiempo. Se observa además que la caída en la creación de sociedades laborales ha tenido una mucha mayor intensidad que la experimentada por las otras formas jurídicas (2,4\% han sido las creadas en el periodo 2015-17) frente a un $13,5 \%$ de cooperativas, y $8,9 \% \%$ de sociedades anónimas y limitadas. 


\section{Tabla 4. Sociedades de corte IT-TIC (CNAEs 26, 61, 62, 631) creadas en España desde el año 2000 al 2017}

\begin{tabular}{|l|c|c|c|c|c|c|}
\hline & \multicolumn{2}{|c|}{ SAy SL } & \multicolumn{2}{c|}{ SCOOP } & \multicolumn{2}{c|}{ SLAB } \\
\hline & $\begin{array}{c}\text { \% con respecto } \\
\text { al total del } \\
\text { periodo 2000- } \\
2017\end{array}$ & No empresas & $\begin{array}{c}\text { \% con respecto } \\
\text { al total del } \\
\text { periodo 2000- } \\
2017\end{array}$ & No empresas & $\begin{array}{c}\text { \% con respecto } \\
\text { al total del } \\
\text { periodo 2000- } \\
2017\end{array}$ & No empresas \\
\hline $\mathbf{2 0 0 0 - 2 0 0 4}$ & $37.4 \%$ & 11.551 & $45.9 \%$ & 17 & $56,0 \%$ & 280 \\
\hline $\mathbf{2 0 0 5 - 2 0 0 9}$ & $28.2 \%$ & 8.731 & $10.8 \%$ & 4 & $25,8 \%$ & 129 \\
\hline $\mathbf{2 0 1 0 - 2 0 1 4}$ & $25.5 \%$ & 7.889 & $29.7 \%$ & 11 & $15,8 \%$ & 79 \\
\hline $\mathbf{2 0 1 5 - 2 0 1 7}$ & $8.9 \%$ & 2.748 & $13.5 \%$ & 5 & $2,4 \%$ & 12 \\
\hline Total & $100.0 \%$ & 30.919 & $100.0 \%$ & 37.00 & $100,0 \%$ & 500.00 \\
\hline
\end{tabular}

SA: Sociedades anónimas; SL: sociedades limitadas, excluidas las laborales; SCOOP: sociedades cooperativas; SLAB: sociedades laborales

FUENTE: Elaboración propia a partir de la base de datos SABI.

Conviene apuntar que esta pérdida de peso en la creación de sociedades de economía social no se ciñe al ámbito IT-TIC, sino que es visible en el conjunto de la economía (tabla 3), aunque se observa, como ya apuntábamos, de forma un poco más acusada.

Habría que preguntarse cuál es la razón de esta pérdida de interés por la forma cooperativa 0 laboral a la hora de crear empresas de corte tecnológico. ¿Se debe al desconocimiento por la misma por parte de los emprendedores, o tiene sus raíces en razones de base económica, jurídica o social? ¿Puede ser por un rechazo a la toma de decisiones democrática, a la distribución de beneficios en función de la actividad, a razones fiscales, de tipo jurídico,...?

Al objeto de arrojar algo de luz a estas cuestiones, hemos extraído de la base de datos SABI información económico-financiera de estas entidades, en los sectores IT-TIC analizados, para ver si existen diferencias en los indicadores o en las tendencias entre las formas jurídicas, que puedan sustentar la falta de motivación por las fórmulas de la economía social, lo cual analizaremos en el siguiente apartado. 


\section{Empresas de corte tecnológico de economía social y de capital. Diferencias en sus indicadores económico-financieros}

En primer lugar, y en el ámbito del tamaño, cabe indicar que las sociedades anónimas y limitadas (excluidas las laborales) tienen una distribución por tamaños más equilibrada (un 21\% facturan por encima de los 5 millones de euros, un 44\% entre 1 y 5 millones, y en un 35\% no superan el millón de euros). Por el contrario, las cooperativas y las sociedades laborales están mucho más polarizadas: las cooperativas en su mayor parte son sociedades de mayor dimensión (un 67\% facturan más de 5.000.000 euros), y el resto no exceden el millón, y las sociedades laborales son mayoritariamente de pequeña dimensión (49\%) o de mediana dimensión (49\%), y solo un $2 \%$ facturan por encima de los 5 millones de euros.

\section{Tabla 5. Sociedades de corte IT-TIC (CNAEs 26, 61, 62, 631) por tamaño en el año 2017 (a partir de SABI)}

\begin{tabular}{|c|c|c|c|c|c|c|}
\hline $\begin{array}{c}\text { Facturación } \\
\text { (miles de euros) }\end{array}$ & SAy SL & SCOOP & SLAB & SAy SL & SCOOP & SLAB \\
\hline Mas de 5000 & 1.213 & 6 & 1 & $21 \%$ & $67 \%$ & $2 \%$ \\
\hline $\begin{array}{c}>1000 y< \\
5000\end{array}$ & 2.590 & 0 & 25 & $44 \%$ & $0 \%$ & $49 \%$ \\
\hline$>500 y<1000$ & 2.029 & 3 & 25 & $35 \%$ & $33 \%$ & $49 \%$ \\
\hline Total & 5.832 & 9 & 51 & $100 \%$ & $100 \%$ & $100 \%$ \\
\hline
\end{tabular}

SA: Sociedades anónimas; SL: sociedades limitadas, excluidas las laborales; SCOOP: sociedades cooperativas; SLAB: sociedades laborales.

El contraste de algunos de los indicadores económicos más utilizados entre las distintas formas jurídicas en el ámbito IT-TIC en el año 2017 arroja los siguientes resultados (tabla 6):

- Todas las variables relacionadas con la dimensión (ingresos de explotación, número de empleados, volumen de activos, fondos propios, o incluso los resultados), son mayores -tanto la media como la mediana- en las cooperativas (aunque debemos apuntar que solo hay 9), seguidas de las sociedades anónimas y limitadas, siendo las de tamaño más reducido las 
sociedades laborales. Así, por citar uno de ellos, si bien la mediana del volumen de activo en cooperativas era en 2017 cercana a los 7 millones de euros, en sociedades anónimas ascendía a 1,1 millones, quedando la de las sociedades laborales en 631 mil euros.

- En cuanto a la rentabilidad, los resultados no apuntan diferencias claras. Comparando las medianas, ya que los datos que ofrecen las medias están más afectados por los valores extremos, observamos que la rentabilidad económica o rentabilidad de las inversiones en activos de la empresa, es algo superior en cooperativas $(6,58 \%)$ que en sociedades anónimas y limitadas $(5,89 \%)$ y ésta a su vez superior a la obtenida por las sociedades laborales $(5,37 \%)$. Y, de hecho, este mismo orden de prelación se manifiesta en el porcentaje de empresas con rentabilidad positiva, que es del $88,9 \%$ en cooperativas, del $85,6 \%$ en sociedades anónimas $y$ limitadas y del $84 \%$ en laborales.

\section{Tabla 6. Indicadores económicos de las sociedades de corte IT-TIC en 2017}

\begin{tabular}{|l|c|c|c|c|c|c|}
\hline & SA y SL (excluidas laborales) & \multicolumn{2}{|c|}{ S COOP } & \multicolumn{2}{c|}{ S LAB } \\
\hline & Media & Mediana & Media & Mediana & Media & Mediana \\
\hline $\mathrm{N}$ & 6.029 & 6.209 & 9 & 9 & 51 & 51 \\
\hline $\begin{array}{l}\text { Ingresos de explotación } \\
\text { (miles de euros) }\end{array}$ & 13.290 & 1.413 & 34.535 & 7.628 & 1.260 & 1.022 \\
\hline \begin{tabular}{l} 
Num. empleados \\
\hline
\end{tabular} & 64,83 & 15,00 & 176,11 & 134,00 & 15,49 & 12,00 \\
\hline $\begin{array}{l}\text { Resultado del ejercicio } \\
\text { (miles de euros) }\end{array}$ & 752,03 & 51,37 & $2.374,74$ & 404,75 & 65,48 & 27,98 \\
\hline $\begin{array}{l}\text { Activo } \\
\text { (miles de euros) }\end{array}$ & 22.219 & 1.146 & 37.563 & 7.294 & 953 & 631 \\
\hline $\begin{array}{l}\text { Fondos Propios (miles } \\
\text { de euros) }\end{array}$ & 10.599 & 454,68 & 21.157 & 2.572 & 522,98 & 302,66 \\
\hline $\begin{array}{l}\text { Rentabilidad económica } \\
\text { (\%) }\end{array}$ & 8,06 & 5,89 & 2,88 & 6,58 & 8,50 & 5,37 \\
\hline $\begin{array}{l}\text { \% de empresas con Rent. } \\
\text { Ec>0 }\end{array}$ & $-54,82$ & 16,58 & 19,62 & 13,96 & 16,61 & 14,97 \\
\hline $\begin{array}{l}\text { Rentabilidad financiera } \\
\text { (\%) }\end{array}$ & \multicolumn{2}{|c|}{$85,6 \%$} & $88,9 \%$ & & \\
\hline
\end{tabular}




\begin{tabular}{|c|c|c|c|c|c|c|}
\hline $\begin{array}{l}\% \text { de empresas con Rent } \\
\text { fin }>0\end{array}$ & \multicolumn{2}{|c|}{$86,6 \%$} & \multicolumn{2}{|c|}{$100 \%$} & \multicolumn{2}{|c|}{$82,4 \%$} \\
\hline $\begin{array}{l}\% \text { de empresas con Rent } \\
\text { fin }>10 \%\end{array}$ & \multicolumn{2}{|c|}{$61,8 \%$} & \multicolumn{2}{|c|}{$66,7 \%$} & \multicolumn{2}{|c|}{$60,8 \%$} \\
\hline $\begin{array}{l}\% \text { de empresas con Rent } \\
\text { fin }>20 \%\end{array}$ & \multicolumn{2}{|c|}{$44,9 \%$} & \multicolumn{2}{|c|}{$33,3 \%$} & \multicolumn{2}{|c|}{$33,3 \%$} \\
\hline Liquidez general & 3,77 & 1,63 & 2,20 & 1,92 & 2,39 & 1,89 \\
\hline$\%$ de Liq gen > 1,5 & \multicolumn{2}{|c|}{$55 \%$} & \multicolumn{2}{|c|}{$88,9 \%$} & \multicolumn{2}{|c|}{$68,6 \%$} \\
\hline Endeudamiento (\%) & $59,25 \%$ & $56,20 \%$ & $65,12 \%$ & $59,32 \%$ & $51,73 \%$ & $48,68 \%$ \\
\hline $\begin{array}{l}\% \text { de empresas con End. } \\
>60 \%\end{array}$ & \multicolumn{2}{|c|}{$84,1 \%$} & \multicolumn{2}{|c|}{$80 \%$} & \multicolumn{2}{|c|}{$64,5 \%$} \\
\hline
\end{tabular}

FUENTE: Elaboración propia a partir de SABI.

- En cambio, si analizamos la rentabilidad financiera, o rentabilidad final del socio, son las cooperativas las que arrojan la menor mediana (13,9\%), por detrás de las laborales $(14,97 \%)$ y de las anónimas y limitadas (16,58\%). De hecho, si bien el porcentaje de empresas con rentabilidad positiva es superior en cooperativas, ya que son el $100 \%$, frente a un $86 \%$ y $82 \%$ en anónimas/limitadas y laborales respectivamente, se aprecia que las sociedades anónimas y laborales presentan en general mayores rentabilidades financieras. De hecho, el 44,9\% presentan una rentabilidad superior al $20 \%$, porcentaje muy superior al $33 \%$ que presentan las fórmulas de economía social.

- $\quad$ En cuanto a liquidez, se observa una mayor liquidez en las sociedades cooperativas y laborales (mediana de 1,91 y 1,89) frente a las anónimas y limitadas (mediana 1,63), y este orden de prelación se manifiesta igualmente si cuantificamos el porcentaje de empresas que tienen una liquidez por encima de 1,5, lo que se considera con carácter general como aceptable $(88,9 \%$ de las cooperativas, $68,6 \%$ de las laborales y 55\% de las anónimas y limitadas).

- $\quad$ En cuanto al endeudamiento, es algo superior en cooperativas $(59,32 \%)$ que en anónimas y limitadas $(56,20 \%)$, y en ambos casos muy superior al de las laborales $(48,68 \%)$, orden de prelación idéntica al observado en los indicadores de tamaño, ya que es comprensible que aquellas entidades de mayor dimensión hayan requerido de un mayor volumen de financiación ajena para financiar ese crecimiento. En ambas sociedades, anónimas/limitadas y cooperativas, el porcentaje de empresas con endeudamiento superior al $60 \%$, supera al $80 \%$.

Por último, nos resta analizar cómo han evolucionado las distintas tipologías empresariales en los últimos 5 años (tabla 6), ya que más allá de la foto fija de 2017, es interesante ver la respuesta que están dando a los retos del momento. 
Cabe destacar que son las sociedades anónimas y limitadas y las laborales las que muestran un mayor incremento de actividad, visible en términos de facturación y de inversiones en activos. En cuanto a la facturación, creció en un $76,3 \%$ de las sociedades anónimas/limitadas y en el $70 \%$ de las laborales, (con una tasa del $36 \%$ las primeras y del $39 \%$ las segundas), por delante de las cooperativas, que aumentaron la facturación en un $66,7 \%$ de las entidades, siendo la mediana de su tasa de crecimiento del $8,8 \%$. Las inversiones en activos siguen una evolución similar, crecen en sociedades anónimas y laborales (en el 75,5\% y el 72,7\% de las entidades respectivamente), y en menor cuantía en cooperativas, que solo se ven incrementadas en el $50 \%$ de las entidades.

Sin embargo, el orden es inverso si analizamos los resultados empresariales y la rentabilidad, tanto económica como financiera). Así, el $66 \%$ de las cooperativas mejoran sus resultados empresariales en este periodo, siendo la tasa mediana de crecimiento del $26 \%$, cuando en sociedades anónimas/ limitadas y en laborales, más de la mitad de las empresas minoran sus resultados $(51,6 \%$ en anónimas/limitadas, y $55,6 \%$ en laborales), siendo la tasa mediana de crecimiento negativa en ambos casos (-5,9\% y - 20,5\% respectivamente). Los indicadores de rentabilidad siguen una evolución similar. En sociedades cooperativas mejora la rentabilidad económica en el $66,7 \%$ de las empresas, y la financiera en el 83,3\%, mientras que tan solo lo hace en un $39,9 \%$ y $37,1 \%$ de las anónimas/limitadas y en un $33,3 \%$ y $26,7 \%$ de las laborales.

En cuanto a la liquidez general, en el periodo analizado, aumenta en un $53,7 \%$ de las sociedades anónimas/limitadas, y por el contrario se reduce en un $66 \%$ de las cooperativas y en un $56 \%$ de las sociedades laborales. No obstante, pese a esta evolución, recordemos que en 2017 las sociedades cooperativas y las laborales presentaban una mayor liquidez que las anónimas/limitadas. Y en cuanto al endeudamiento, han sido las cooperativas las que han sufrido un mayor incremento del nivel de endeudamiento, visible en el $50 \%$ de las entidades, lo que como ya hemos indicado puede deberse a sus mayores necesidades de financiación, dado su mayor dimensión media empresarial. 
Tabla 7. Evolución de las sociedades con actividad TIC en el periodo 2007-16 y 12-16, según su forma jurídica

\begin{tabular}{|l|c|c|c|c|c|c|c|c|c|c|c|c|}
\hline & \multicolumn{3}{|c|}{ Sociedades anónimas y limitadas } & \multicolumn{3}{|c|}{ Sociedades cooperativas } & \multicolumn{3}{|c|}{ Sociedades laborales } \\
\hline & $\begin{array}{c}2012 \\
\text { Medna }\end{array}$ & $\begin{array}{c}2016 \\
\text { Medna }\end{array}$ & $\begin{array}{c}\text { Var } \\
12-16 \\
\text { medna }\end{array}$ & $\begin{array}{c}\% \text { con } \\
\text { var }+\end{array}$ & $\begin{array}{c}2012 \\
\text { Medna }\end{array}$ & $\begin{array}{c}2016 \\
\text { Medna }\end{array}$ & $\begin{array}{c}\text { Var } \\
12-16 \\
\text { medna }\end{array}$ & $\begin{array}{c}\% \text { con } \\
\text { var }+\end{array}$ & $\begin{array}{c}2012 \\
\text { Medna }\end{array}$ & $\begin{array}{c}2016 \\
\text { Medna }\end{array}$ & $\begin{array}{c}\text { Var } \\
12-16 \\
\text { medna }\end{array}$ & $\begin{array}{c}\% \text { con } \\
\text { var + }\end{array}$ \\
\hline I exp & 1048.6 & 1262.4 & $36.5 \%$ & 76.3 & 13819.9 & 5119.0 & $8.8 \%$ & 66.7 & 593.0 & 817.2 & $39.0 \%$ & 70.5 \\
\hline Rdo ej & 15.7 & 38.2 & $-5.9 \%$ & 48.4 & 133.6 & 61.7 & $26.0 \%$ & 66.7 & 4.2 & 17.0 & $-20.5 \%$ & 44.4 \\
\hline Activo & 836.9 & 1020.2 & $35.2 \%$ & 75.5 & 16156.6 & 5139.3 & $-1.7 \%$ & 50.0 & 465.8 & 569.3 & $32.6 \%$ & 72.7 \\
\hline F. prop & 299.7 & 384.4 & $35.9 \%$ & 73.8 & 8323.8 & 1379.7 & $7.2 \%$ & 66.7 & 182.3 & 271.2 & $28.4 \%$ & 77.8 \\
\hline $\begin{array}{l}\text { Rent. } \\
\text { ec }\end{array}$ & 2.606 & 5.1 & $-36.4 \%$ & 39.9 & 0.2 & 4.0 & $474.1 \%$ & 66.7 & 1.5 & 3.1 & $-81.7 \%$ & 33.3 \\
\hline $\begin{array}{l}\text { Rent. } \\
\text { fin }\end{array}$ & 8.844 & 15.1 & $-43.9 \%$ & 37.1 & 0.7 & 10.5 & $394.3 \%$ & 83.3 & 3.0 & 10.5 & $-80.0 \%$ & 26.7 \\
\hline Liq gen & 1.621 & 1.6 & $4.2 \%$ & 53.7 & 2.9 & 1.7 & $-11.2 \%$ & 33.3 & 2.0 & 2.0 & $-11.2 \%$ & 44.4 \\
\hline R. End & 59.9 & 57.9 & $-6.3 \%$ & 40.8 & 62.5 & 59.7 & $0.7 \%$ & 50.0 & 54.8 & 52.1 & $-6.7 \%$ & 37.8 \\
\hline
\end{tabular}

\% con var +: \% de empresas con variación positiva; medna: mediana

FUENTE: Elaboración propia a partir de SABI.

Todos estos índices y su evolución no permiten sostener que la limitada presencia de figuras societarias propias de la Economía social en sectores tecnológicos de la llamada economía digital se deba a razones de base económica ya que no observamos diferencias relevantes, especialmente si nos referimos a los resultados alcanzados por estas. Ello puede apuntar más bien al desconocimiento de estas figuras societarias por los jóvenes emprendedores, escaso atractivo para ellos de las mismas o la mayor tradición y prevalencia hasta la fecha en sectores de tecnología avanzados de las figuras de societarias de capital tradicionales (S.L. y S.A) (Bel y Lejarriaga, 2018). 


\section{Discusión. Hacia una economía del conocimiento con valores como objetivo}

Las sociedades más avanzadas en el mundo son aquellas que se diferencian por hacer un mayor uso del conocimiento en su economía. En el informe ABACO de la Fundación BBVA-IVIE (observatorio de actividades basadas en el conocimiento) (IVIE 2013), se observa claramente cómo los países con economías más desarrolladas, pero también con mayores cuotas de bienestar, suelen ser aquellos que tienen a su vez un mayor peso de las actividades basadas en el conocimiento en porcentaje de su PIB, como es el caso de EE. UU, Reino Unido, Países Bajos, Finlandia y Dinamarca. De hecho, el crecimiento empresarial de estos países se ha producido haciendo un mayor uso del conocimiento, siendo hoy el peso de dichas actividades superior al $70 \%$ en sus economías. En las últimas décadas estas actividades han crecido igualmente en otros países, aunque por debajo de esa cifra, como es el caso de España, cuyo porcentaje se sitúa próximo al $60 \%$.

Sin embargo, hay que reconocer que existen Comunidades Autónomas en España que ya se sitúan en el 60-70\%, destacando particularmente el caso del País Vasco, Comunidad Autónoma que mejor ha resistido en España la crisis económica de estos años, y que ha seguido una pauta diferente al resto de España, manteniendo su gasto en I+D, apostando por la innovación y el emprendimiento, en el que ha adquirido especial relieve el acometido bajo formas de economía social. De hecho, cabe mencionar que un pilar muy potente en su economía y su tejido productivo es un grupo de Economía Social, Mondragón Corporación Cooperativa (MCC) (Ucin, 2019).

Con todo, el avance en la economía del conocimiento es un reto estratégico para España, al igual que para todos los países avanzados. Y ello hace necesario un sistema educativo cada vez más desarrollado, ya que como señalan algunos autores "el avance del uso productivo del conocimiento en España requiere de esfuerzos combinados del sistema educativo y del sistema productivo, así como de las políticas públicas" (Pérez y Benages, 2012).

Tras la grave crisis global que siguió a la caída de Lehman Brothers, se impuso una obligada reflexión de las causas que la motivaron. En el Congreso Internacional de CIRIEC celebrado en el 2008 en Sevilla, Joseph Stiglitz, Nobel de Economía, ya señaló que más allá de los factores desencadenantes de la misma (las hipotecas subprime, el sector inmobiliario, etc.,), había que buscar como origen de la crisis la pérdida de valores en la economía, derivada de un fundamentalismo de mercado que falló. Así, aunque hoy por hoy no se discuten los paradigmas de la economía de mercado, la clave estaría en recuperar y fomentar una economía sostenible con valores, que atenúe los fallos que en un mercado escasamente regulado puede producir la ausencia de valores (Stiglitz, 2008). 
La Unión Europea afortunadamente parece que comparte este diagnóstico, ya que en su Horizonte 2020 y, más concretamente en el programa RIS3, en la llamada estrategia de especialización inteligente se destaca la voluntad de propiciar un crecimiento económico inteligente, sostenible e integrador, lo que coincide a la perfección con este discurso. Hoy la Unión Europea, como señalan sus responsables políticos y técnicos, apoya acciones concretas, para impulsar el desarrollo de la Economía Social (Katainen, Thyssen, Bienkowska, 2019) (Engelmann, 2019).

La economía debe afrontar dos retos importantísimos: la innovación basada en el conocimiento y la sostenibilidad, aunque en ambos casos su enfoque debe ser amplio, y abarcar más allá del sentido estrictamente económico. De hecho, en cuanto al primero de ellos, ya en la introducción del informe de COTEC de 2016, se definía la innovación como "todo cambio (no solo tecnológico), basado en el conocimiento (no solo científico), que genera valor (no solo económico)", haciendo una clara referencia al valor social. En cuanto al segundo reto la sostenibilidad, debe también considerarse en un sentido amplio, en términos de sostenibilidad económica, social y medio ambiental. Además, ambos retos están claramente conectados, ya que una innovación derivada del conocimiento y los valores es una innovación sostenible. Y su implantación requiere de una apuesta por el conocimiento, y de su mano de la investigación, pero, también, de una adecuada formación para transmitirlo con una cultura en valores.

En este sentido, si vemos nuestra política presupuestaria y comportamiento empresarial de los últimos años, no podemos afirmar que en general éstos se hayan orientado siempre al logro de estos objetivos. Ciertamente, si observamos la evolución del gasto en $\mathrm{l}+\mathrm{D}$ de los últimos años, constatamos que si bien en el año 2010 España invertía el 1,4\% del PIB en l+D, en el año 2018, este porcentaje apenas llegaba al 1,2\%, lo que representa una pérdida de más del $20 \%$ de la cuantía destinada a la l+D. España está hoy a la mitad del gasto, en términos de porcentaje del PIB, de la media de la OCDE, lo que denota un déficit importantísimo. Afortunadamente, desde la Administración española se reitera ahora constantemente este discurso, lo que hace prever un cambio de sentido en los futuros Presupuestos Generales del Estado.

Del mismo modo, fortalecer el vector del conocimiento pasa también por destinar más recursos a educación, en todos sus niveles, y especialmente a las Universidades. La media de gasto destinado a Universidades en España es del 1,28\% del PIB, a diferencia del 1,56\% de la media de la OCDE, un $18 \%$ menos (CYD 2017), y como ocurre en investigación, se observa una evolución negativa en gasto por estudiante, una disminución del 13\% de 2010 a 2015, frente a un crecimiento medio en la OCDE del $5 \%$ en el mismo periodo, siendo España el segundo país de la OCDE que más lo redujo (Hernández y García 2018).

La respuesta desde la formación a ambos retos (innovación y sostenibilidad), pasa por no olvidar algunas de las debilidades de nuestro sistema educativo, destacadas en algunos informes. La consultora KPMG, preguntaba a los egresados su opinión sobre la formación recibida, a lo que respondían, 
en términos generales de forma positiva, pero reivindican una mayor formación de carácter práctico, y una mayor conexión con el tejido productivo (KPMG, 2017). Por otro lado, el informe EVERIS recoge la opinión de los empleadores acerca de la formación impartida en las Universidades españolas, la cual ven adecuada, pero necesitada de una mejora de las soft skills. Y a estos retos debemos añadirle uno más, en el que ningún informe insiste, que no es otro que la necesidad de formar en valores, algo necesario si se quiere extender, como Europa parece pretender, la economía y el emprendimiento social para avanzar en una sociedad no solo más inteligente, también más equitativa e inclusiva (EVERIS, 2017).

Y en esta realidad, la economía social y sus empresas deben sin duda jugar un papel esencial, lo que sin duda implica que sea estratégica su presencia en aquellos sectores de actividad empresarial más alineados con el signo de los tiempos, es decir, los sectores que definen la llamada cuarta revolución industrial, transformación digital, robótica e inteligencia artificial. Es necesaria la emergencia de emprendimiento innovador, tecnológicamente avanzado y con valores, pero ésta se ve dificultada ante el insuficiente alineamiento del mundo de la Economía Social, la Universidad y los nuevos sectores tecnológicos (Bel y Lejarriaga, 2018).

Este es sin duda un problema que debe preocupar ya que la necesidad de contar con este tipo de empresas en sectores clave para la Economía ante el importante reto tecnológico que supone la digitalización puede tener consecuencias ya que el progreso social viene inexorablemente determinado por la competitividad y la sostenibilidad que como venimos señalando necesitan de la presencia en sectores que son hoy verdaderos vectores de crecimiento de empresas con valores. No podemos dejar de señalar que países donde mayor progreso social se registra cuentan también con un mayor índice de desarrollo de empresas de la Economía Social, como son las cooperativas (Dave Grace 2014). Del mismo modo, que también se puede observar un alto nivel en el llamado índice de competitividad digital en los países con mayor progreso social. Es posible que un alineamiento en el avance de las formas de Economía Social con el desarrollo de la llamada economía digital pueda favorecer un entorno más favorable al progreso social. 


\section{Tabla 8. TOP-10 de países por Progreso Social, Desarrollo Coo- perativo y Competitividad Digital}

\begin{tabular}{|c|c|c|c|}
\hline Posición & Índice de Progreso Social (SPI)* & índice de Desarrollo Cooperativo \\
(SPI Ranking)
\end{tabular}

FUENTE: Elaboración propia a partir de Dave Grace Asociates. Global Census on Co-operatives 2014. United Nation's Secretariat. *() Posición en el TOP Mundial del Índice de Progreso Social, **[] IMD Digital World Competitiveness Ranking 2019.

Con este fin resulta de interés una primera aproximación hacia el grado de desarrollo de un sector tecnológico tan importante para el desarrollo de nuestra Economía en el mundo de las empresas de Economía Social. 


\section{Conclusiones. Un balance positivo y mejorable}

El examen realizado en este trabajo en relación con la Economía Social y el desempeño del sistema universitario, en particular en lo relativo a la generación del conocimiento y al emprendimiento, y el análisis de la presencia de empresas de Economía Social en los sectores de actividad tecnológica objeto de estudio, nos permiten formular las siguientes conclusiones:

España fue el primer país de la Unión Europea en reconocer formalmente mediante una Ley a la Economía Social, lo que ha permitido una identificación organizativa de las diferentes figuras jurídicas que conforman hoy la realidad económica y social del sector, y ha permitido tomar conciencia del interés de contar con este tipo de empresas en los diferentes sectores de nuestra actividad económica.

Actualmente España es uno de los países de la Unión Europea donde mayor peso tiene la Economía Social contando con firmas líderes en diferentes sectores de su Economía (agroalimentario, crédito, consumo, seguros...), si bien es cierto que, en un economía global y competitiva, la dimensión de la mayor parte de empresas de Economía Social es en general todavía reducida, lo que merma sus posibilidades de innovación y su competitividad a la hora de posicionarse mejor en los mercados.

Todo ello ha contribuido a que la Economía Social goce de un amplio reconocimiento social que alcanza también al mundo académico. Buena muestra de ello es el hecho de que en las últimas décadas, se han incrementado los trabajos científicos publicados en estos temas, algo a lo que han contribuido dos revistas científicas impulsadas desde el mundo universitario, CIRIEC-España, editada por la asociación científica del mismo nombre, ubicada en la Universitat de València y Revesco, editada por la Escuela de Estudios Cooperativos de la Universidad Complutense de Madrid, ambas indexadas en SCOPUS y ESCI, dos de las bases de datos de revistas científicas más reconocidas.

Por otro lado, el hecho de que hemos dotar a la sociedad de una economía más intensa en conocimiento, capaz de afrontar exitosamente el reto tecnológico que supone la cuarta revolución industrial mediante un emprendimiento innovador pero con valores, que nos permita crecer de una manera social y económicamente sostenible, sin duda pone en valor el papel de una institución como la Universidad, como centro de transmisión y generación de conocimiento y a la vez de fórmulas empresariales como las de la Economía Social, cuyos principios las sitúan al servicio de las personas y de la sociedad. 
Afortunadamente, las universidades españolas contemplan cada día más, de una forma visible dentro de su dimensión social, la necesidad de un mayor compromiso con la valorización del conocimiento que generan y transfieren, participando activamente en la creación de un ecosistema emprendedor e innovador en sus campus. Su desempeño académico es sin duda razonablemente positivo como acredita una correcta lectura e interpretación del posicionamiento de las Universidades españolas en los rankings internacionales universitarios más reconocidos (ARWU, THE y QS), y en especial si hablamos de su desempeño docente e investigador. Pero sin duda puede mejorar y debe hacerlo en lo relativo a la transferencia y generación de conocimiento, así como con una mayor internacionalización de sus actividades académicas.

Igualmente, es cierto que el crecimiento y extensión territorial de las Universidades ha contribuido a una mayor igualdad de oportunidades y el logro de una mayor cohesión social y territorial, algo que debe preservarse como elemento claro de su compromiso social. Además, preocupa también la necesidad de contar en el futuro con perfiles profesionales capaces de afrontar el nuevo reto tecnológico, donde la caída de la demanda de estos estudios puede ser un serio problema que se ve agudizado por la existencia de una brecha social y de género.

Del mismo modo, la constatación de la calidad emprendedora de las iniciativas que emergen en el ecosistema universitario en el sentido de su mostrada mayor capacidad innovadora, mayor uso de tecnologías avanzadas y capacidad de internalización permite abordar mejor los importantes desafíos tecnológicos que supone la nueva economía. Sin embargo, esta idea contrasta con el hecho de que por el momento el uso de las fórmulas de Economía Social es bastante limitado en este ecosistema, si bien se observan iniciativas que tratan de promover este tipo de fórmulas.

Finalmente, se observa que las cooperativas y las sociedades laborales ya están implantándose en un sector tecnológico tan avanzado de la economía digital como son el ámbito IT-TIC si bien el peso de la Economía Social en las empresas que operan en el ámbito de las IT-TIC, es inferior al que encontramos en el conjunto de la economía (solo un 1,62\% de las empresas frente al 1,95\% del conjunto de la economía), aunque esto es principalmente debido al reducido número de cooperativas existentes, ya que las sociedades laborales, aunque con poco peso, se encuentran algo más representadas que en la generalidad de sectores. Esto es consecuencia de que las fórmulas capitalistas han sido siempre las elegidas mayoritariamente en el sector de las IT-TIC, y esta realidad no solo no se ha invertido, sino que se ha visto acrecentada en el periodo 2000-2017, siendo en la actualidad aún más reducido el número de sociedades de economía social creadas en estos sectores, en comparación con las anónimas/limitadas.

Si buscamos las razones de esta no preferencia por las fórmulas de Economía Social, debemos indicar que no residen en factores de tipo económico-financiero, ya que no se han observado diferencias relevantes en sus principales indicadores. De hecho, la rentabilidad, indicador clave para los que deciden crear una empresa, no arroja diferencias significativas entre las diferentes tipologías 
empresariales en 2017, y en lo que a su evolución se refiere, han sido las cooperativas las que mejor evolución en sus resultados y rentabilidad han generado en los últimos 5 años, seguidas de las anónimas y limitadas, y quedando en último lugar las sociedades laborales.

Por todo ello, creemos que no existen razones objetivas para que esta realidad no revierta en positivo con un mayor incremento y presencia de la economía social en este sector, clave para dar una mayor orientación tecnológica a nuestro tejido productivo. Entendemos que un mayor alineamiento de la economía social con las iniciativas de emprendimiento nacidas bajo el ecosistema universitario contribuirán favorablemente hacia una economía más inteligente y con valores, camino inexorable para contar con una economía más competitiva y a la vez sostenible.

\section{Referencias}

ALDÁS, J. (2019): "Cuarta revolución, empleo y universidades", Expansión, 28 de mayo de 2019.

ACADEMIC RANKING OF WORLD UNIVERSITIES (ARWU) (2019): http://www.shanghairanking. com/arwu2019.html

BEL DURÁN, P. y LEJARRIAGA PÉREZ DE LAS VACAS, G. (2018): "Sociedades de responsabilidad limitada calificables y sociedades participadas: una aproximación a su cuantificación". REVESCO, Revista de Estudios Cooperativos, Primer Cuatrimestre, 127, 9-25. DOI: 10.5209/ REVE.59773.

CHAN, K. (2017): "Witch countries provide their citizens with the best higher education?", World Economic Forum, https://www.weforum.org/agenda/2017/05/which-countries-provide-their-citizenswith-the-best-higher-education/.

CHAVES ÁVILA, R., MONZÓN CAMPOS, J.L. (2018): "La economía social ante los paradigmas económicos emergentes: innovación social, economía colaborativa, economía circular, responsabilidad social empresarial, economía del bien común, empresa social y economía solidaria", CIRIEC-España, Revista de Economía Pública, Social y Cooperativa, 93, 5-50, DOI:10.7203/ ClRIEC-E.93.12901.

COTEC (2016): Informe COTEC. Innovación en España, Ed. Fundación COTEC para la innovación, Madrid, pp. 237

CYD (2017): Informe CyD 2016 La contribución de las universidades españolas al desarrollo, Ed. Fundación Conocimiento y Desarrollo, Barcelona, 279 pp. 
DAVE GRACE ASSOCIATES (2014): "Measuring the size and scope of the cooperative economy: Results of the 2014", Global census on cooperatives, United Nation's Secretariat.

DELGADO, L. (2017): Proyecto PRISUE. Posicionamiento y reconocimiento internacional del sistema Universitario Español. Disponible en: https://www.crue.org/Documentos\%20compartidos/Publicaciones/Monograf\%C3\%ADas/RANKING_XavierGrau.pdf.

ENGELMANN, U. (2019): "Conjunto de acciones de la Comisión Europea en favor de la Economía Social", Mediterráneo Económico, colección de estudios socioeconómicos, 32, 423-435.

FLORES, D., GUZMÁN, C. \& BARROSO, M.O. (2015): "La formación en economía social. Análisis de la oferta universitaria de posgrado en España", REVESCO, Revista de Estudios Cooperativos, 121, 89-113. http://dx.doi.org/10.5209/rev_REVE.2016.v121.49703.

HERNÁNDEZ ARMENTEROS, J. y PÉREZ GARCÍA, J.A. (2018): La Universidad Española en Cifras, CRUE Universidades Españolas, 2016/2017, Santander Universidades.

COGECA (2015): Informe COGECA. Development of agricultural cooperatives in the EU, Bruselas.

EVERIS (2017): Informe Fundación EVERIS. III Ranking Universidad-Empresa. Encuesta a las empresas españolas sobre la empleabilidad de los recién titulados.

KATAINEN, J., THYSSEN, M., BIENKOWSKA, E. (2019): "Hacia un crecimiento sostenible y justo: Europa apoya a la Economía Social", Mediterráneo Económico, colección de estudios socioeconómicos, 32, 49-53.

KPMG (2017): Informe KPMG. Transformación y futuro de la Universidad Pública en España.

IVIE (2013): "Observatorio de las actividades basadas en el conocimiento". Informe ABACO, www. observatorioabaco.es

JULIÁ-IGUAL, J.F., MELIÁ-MARTÍ, E., CARNICER-ANDRÉS, P. \& VILLALONGA-GRAÑANA, I. (2014): "El ecosistema universitario de emprendimiento y la economía social", XV Congreso de Investigadores en Economía Social, Santander.

JULIÁ IGUAL, J.F. (2019): "La Universidad Española. Evolución y retos antes una nueva sociedad y economía", Informe España, Ed. Fundación Encuentro, Universidad Comillas.

JULIÁ IGUAL, J.F. y MIRANDA RIBERA, E. (2019): "La Constitución Española y la Economía Social ante el reto de una nueva sociedad", CIRIEC-España, Revista de Economía Pública, Social y Cooperativa, $\mathrm{N}^{\circ}$ extraordinario 2018, 119-126.

JULIÁ IGUAL, J.F., MELIÁ MARTÍ, E., VILLALONGA GRAÑANA, I., CARNICER ANDRÉS, M.åP., (2015): "El emprendimiento en el sistema universitario. El caso de las universidades de la ciudad de Valencia", CISE, Centro Internacional Santander Emprendimiento, Ed. Universidad Cantabria. 
LEJARRIAGA PÉREZ DE LAS VACAS, G. y BEL DURÁN, P. (2018): "Emprendimiento, emprendimiento social, emprendimiento en economía social y emprendimiento a través de organizaciones de participación", V Jornadas de investigación y docencia en materia de empresas de participación. Emprendimiento social y nuevas formas de hacer economía: su relación con las organizaciones de participación y la economía social, 16 de febrero de 2018, Madrid.

MELIÁN NAVARRO, A., CAMPOS I CLIMENT, V. \& SANCHIS PALACIO, J.R. (2017): "La educación de postgrado en Economía Social en la universidad española ¿una asignatura pendiente?", CIRIEC-España, Revista de Economía Pública, Social y Cooperativa, 89, 33-55. DOI: 10.7203/ CIRIEC-E.89.9673.

PALOMO ZURDO, R., FERNÁNDEZ TORRES, Y. y GUTIÉRREZ FERNÁNDEZ, M., (2018): "Banca cooperativa y transformación digital: hacia un nuevo modelo de relación con sus socios y clientes", REVESCO, Revista de Estudios Cooperativos, 129, 161-182. DOI: 10.5209/REVE.62490.

PÉREZ, F. y BENAGES, E. (2012): "EI PIB basado en el conocimiento", Observatorio ABACO.

QS Employability: https://www.topuniversities.com/university-rankings/employability-rankings/2018

PÍRIZ (2017): "El largo camino hacia la calidad. Algunas reflexiones sobre la Universidad Española", Nueva Revista de Cultura, Política y Arte, UNIR, 163, 8-27.

PASTOR, J.M. (Dir.) (2019): La contribución socioeconómica del Sistema Universitario Español, Ed. IVIE.

SÁNCHEZ ESPADA, J., MARTíN LÓPEZ, S., BEL DURÁN, P., LEJARRIAGA PÉREZ DE LAS VACAS, G. (2018): "Educación y formación en emprendimiento social: características y creación de valor social sostenible en proyectos de emprendimiento social", REVESCO, Revista de Estudios Cooperativos, 129, 16-38. DOI: 10.5209/REVE.62492.

SEGUÍ MAS, E., OLTRA, V., TORMO CARBÓ, G. \& SARRIÓN VIÑES, F. (2017): "Rowing against the wind: how do times of austerity shape academic entrepreneurship in unfriendly environments?", Int Entrep Manag J, https://doi.org/10.1007/s11365-017-0478-z.

STIGLITZ, J. (2009): "Moving beyond Market Fundamentalism to a More Balanced Economy", Annals of Public and Cooperative Economics, 80:3, 345-360, https://doi.org/10.1111/j.14678292.2009.00389.x. Opening session of the 27th international congress of CIRIEC, Sevilla 22-24 September, 2008.

Times Higher Education Europe Teaching Rankings 2018: https://www.timeshighereducation.com/ rankings/europe-teaching/2018\#!/page/0/length/25/sort_by/rank/sort_order/asc/cols/undefined.

UCIN, I. (2019): "Mondragón. Una experiencia cooperativa innovadora e internacional", Mediterráneo Económico, colección de estudios socioeconómicos, 32, 169-177. 
